\title{
An Unusual Presentation of Advanced Pancreatic Cancer: Coeliac Axis Occlusion and Acute Upper Gut Ischemia
}

\author{
Hilmi D. Elsiddig ${ }^{1}$, Ibrahim O. Jabra², A.A. Dabora ${ }^{3}$, Shakir Z. Ibrahim ${ }^{1}$
}

Corresponding author:

Hilmi D. Elsiddig, MD

Assistant professor of surgery

University of Khartoum

Faculty of Medicine

Department of Surgery

P.0 Box 8081 Khartoum, Alamarat

Sudan

E-mail: hilmidaoud@gmail.com

\section{Abbreviations:}

CT: Computed Tomography scan SMA: Superior Mesenteric Artery
'Department of General Surgery, University of Khartoum, Royal Care International Hospital, Sudan

${ }^{2}$ Department of General Surgery, Royal Care International Hospital, Sudan

${ }^{3}$ Department of Hepatobiliary Surgery, Royal care International Hospital, Sudan

\section{ABSTRACT}

We present a case of a fifty-year-old male with acute thrombosis of the celiac trunk secondary to advanced pancreatic cancer. He was admitted to the emergency department with a 4-day history of upper abdominal pain and coffee ground vomitus. Abdominal examination showed signs of generalized peritonitis. CT abdomen was done without contrast because of impaired renal function. The findings consisted of a large pancreatic neck and body mass, fat stranding, free fluid and distended bowel loops. Emergency laparotomy revealed a voluminous pancreatic mass arising from the neck and body of the pancreas, with the coeliac trunk being completely infiltrated by the tumour. A gangrenous stomach and lower oesophagus with big gastric perforation were also noted. The patient also had extensive splenic infarction, however the liver was normal. He became unstable during surgery and nothing could be done. He did not recover from anaesthesia and died in the ICU after 24 hours.

Key words: coeliac-axis occlusion gut ischemia pancreatic cancer

\section{INTRODUCTION}

Coeliac artery thrombosis with ischemia is a rare condition associated with risk factors for thrombosis or embolism. The manifestation is rare because of the large number of collaterals between the coeliac and superior mesenteric artery. Advanced pancreatic cancer maybe a cause of gradual occlusion associated with collaterals, which require special attention during pancreaticoduodenectomy. To the best of our knowledge, acute thrombosis of the coeliac axis associated with acute upper gut ischemia in this clinical setting has not been reported yet.

\section{CASE PRESENTATION}

A 50-year-old male was admitted with 4-day history of acute upper abdominal pain, abdominal distension and coffee grown vomitus. He was tachycardic and hypotensive on admission and abdominal examination showed signs of generalised peritonitis. He had a history of marked weight loss, recurrent upper abdominal pain and dyspepsia for the last eight months. 
Oesophagogastroduodenoscopy was done twice and the finding was not conclusive. CT abdomen was not requested by the treating physician during his illness. On admission, he was dehydrated with acute renal impairment (His serum creatinine was $3.8 \mathrm{mg} / \mathrm{dl}$ and blood urea was $120 \mathrm{mg} / \mathrm{dl}$ ). The nasogastric tube output in the ER was four litres, consisting of a dark fluid. While resuscitating the patient and preparing him for an emergency laparoscopy, CT abdomen (without contrast because of impaired renal function) was done which showed a mass in the pancreatic neck and body, fat stranding, free fluid and distended bowel loops (fig. 1). Emergency laparotomy revealed a gangrenous stomach and lower oesophagus with as well as a gastric perforation with a diameter of $8.5 \mathrm{~cm}$. He also had extensive splenic infarction (fig. 2). There was normal blood flow to the liver, duodenum and small bowel. The intraoperative examination confirmed the presence of the large pancreatic mass involving the neck and body. The coeliac trunk was completely encapsulated by the tumour. The patient was in septic shock and had severe hypotension during the operation. Nothing could be done intraoperatively to help the patient, he was hemodynamically unstable and had extensive infarction. The abdominal cavity was drained with large-bore drain tubes and the abdomen was closed. He did not recover from Anaesthesia and he died in the ICU after 24 hours.

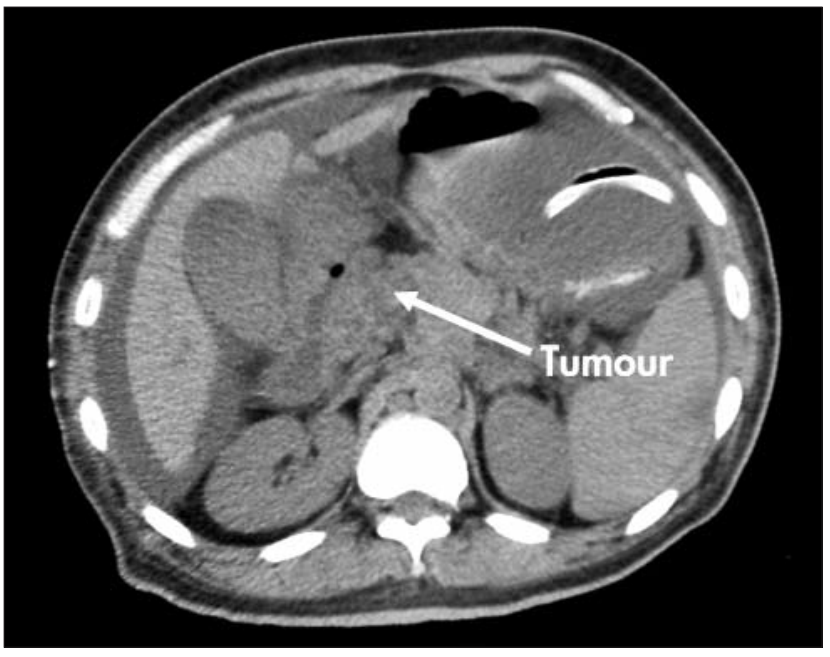

Figure 1 - CT abdomen without contrast showing pancreatic neck and body mass, fat stranding, free fluid and distended bowel loops

\section{DISCUSSION}

Stenosis of the celiac artery is present in up to $10 \%$ of patients undergoing pancreaticoduodenectomy, as reported in series where arteriography was routinely performed before surgery. However, this has no clinical significance, owing to collateral pathways that develop from the SMA via the inferior pancreaticoduodenal
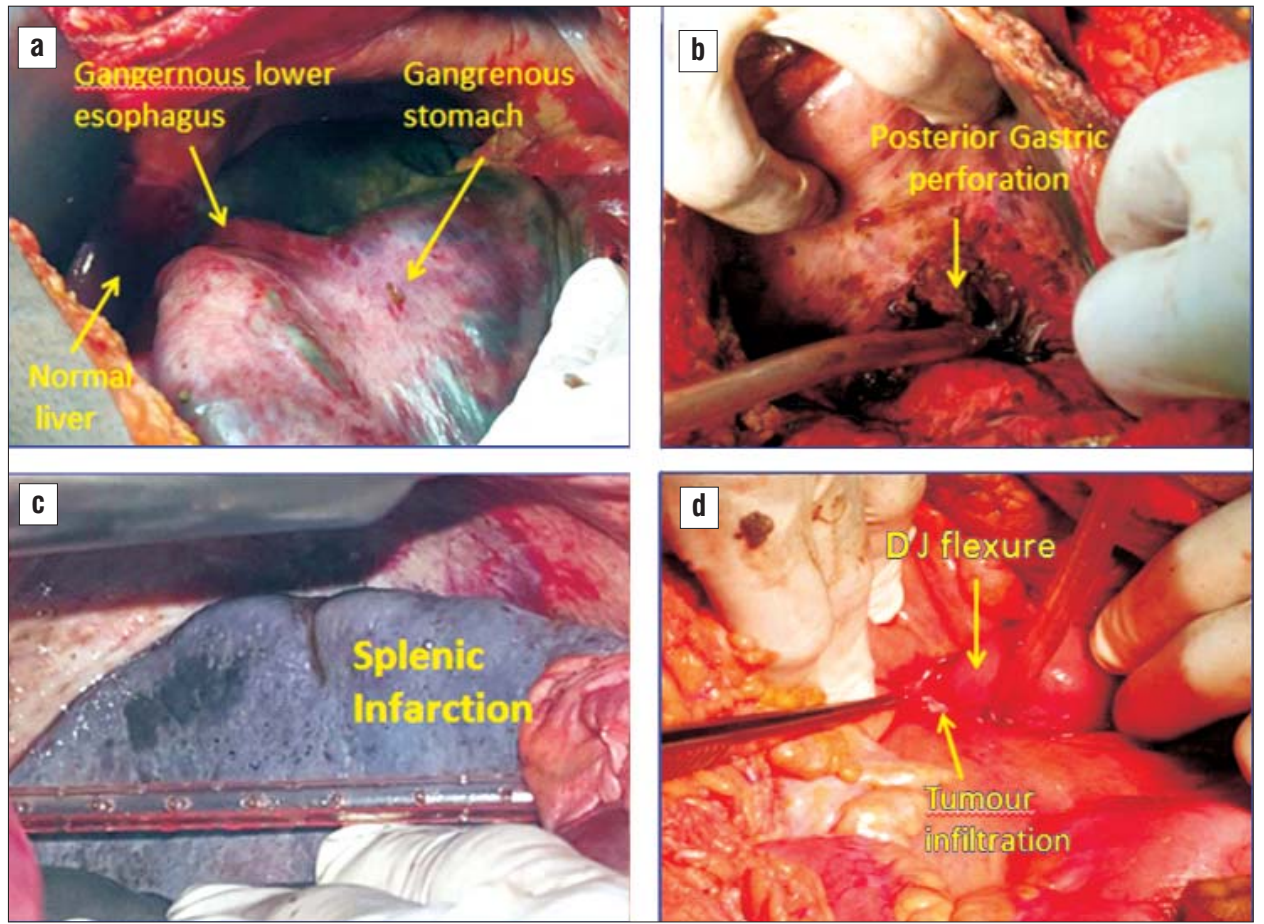

Figure 2 - Intraoperative photos. (a) Gangrenous stomach and lower esophagus; (b) Posterior Gastric perforation; (c Splenic infarction; (d) Tumour infiltrating the root of mesentery-superior mesenteric vessels. DJ - means duodeno-jujenal flexure 
artery to provide retrograde flow through the gastroduodenal artery. Z. Soonawalla et al (1) addressed the importance of identifying these vascular anomalies during pancreatectomy, followed by proper reconstructive procedures. Few cases of acute coeliac artery thrombosis with gastric ischemia and splenic infarction have been reported in the literature, and most of them were caused by myeloproliferative and thrombotic disorders (2). Watson et al (3) reported a case of acute thrombosis of the coeliac trunk secondary to alcohol-induced pancreatitis. The patient received an emergency open thrombectomy. In our case, the patient presented with acute abdominal pain, he had undiagnosed advanced pancreatic cancer with invasion of the coeliac artery and laparotomy showed gastric ischemia and splenic infarction. The ischemia extended up to the lower oesophagus and the patient was unstable during surgery, so there was no rationale for a major resection which would entail a prolonged procedure.
This is probably the first case report of acute coeliac artery occlusion and upper gut ischemia secondary to locally advanced pancreatic cancer. A high index of suspicion, which would entail performing a CT abdomen with intravenous contrast, is mandatory in patient with advanced pancreatic cancer presenting with acute upper abdominal pain.

\section{Conflict of Interest}

The authors declare that there is no conflict of interests regarding the publication of this paper.

\section{REFERENCES}

1. Soonawalla Z, Ganeshan A, Friend P. Celiac artery occlusion encountered during pancreatic resection: a case report. Ann R Coll Surg Engl. 2007 Jan;89(1):W15-7.

2. Saravana K, Aainal AA, Lee SK. Coeliac artery thrombosis: An uncommon cause of an acute abdomen. Med J Malaysia. 2011;66: 273-5.

3. Watson TJ, Childers WK, Campbell J. Acute celiac trunk thrombosis treated with Open Thrombectomy. Ann Vasc Med Res. 2015;2(3): 1023-5. 